\title{
CESR IV PROPOSED TRANSFER LINE DESIGN*
}

\author{
Z. Greenwald, D.L. Rubin \\ Laboratory of Nuclear Studies, Cornell University, Ithaca, NY 14853
}

\begin{abstract}
CESR IV is a proposed dual-bore colliding beam accelerator for B-meson production. It has been designed to be built on the top of the injection synchrotron ring so as to co-exist with the present CESR III ring [1]. In this paper we will describe the design of the CESR IV transfer line. This transfer line splits from the existing transfer line and guides the beam, extracted from the synchrotron, $43 \mathrm{~cm}$ vertically and then injects horizontally into the new ring. The trajectory, taking into account the available space, was determined by horizontal and vertical bending magnets and drift lines. The quadrupoles were optimized to match the TWISS parameters at the input and the output of the transfer line to those of the synchrotron and the new CESR IV ring respectively.
\end{abstract}

\section{INTRODUCTION}

In the CESR IV design [1], the present storage ring and the new one - which will be on top of the synchrotron, will share the synchrotron ring as injector. When designing the new transfer line for CESR IV the following requirements should be considered:

- It will share the input point (coming from the synchrotron) with that of the existing transfer line [2] and will split after $Q T 02$.

- It will have the same beam pipe radius of $1.27 \mathrm{~cm}$ as that of the present transfer line.

- The beam should be guided up to the level of CESR IV, which is $0.43 \mathrm{~m}$ above the synchrotron, and then curved back connecting tangentially to CESR IV.

- TWISS parameters at the input of the transfer line should match those of the synchrotron at S133 and the TWISS parameters at the output of the transfer line should match those of CESR IV at Q051.

- Vertical dispersion should be minimized which requires small bending angles in the vertical direction.

- The new transfer line should fit together with the present one in the available space.

\section{TRANSFER LINE DESIGN}

CESR IV transfer line will share the present transfer line up to $Q T 02$ and then will split off with an angle of $9^{\circ}$. The splitting dipole will be located in the drift line F146 $(2.4 \mathrm{~m})$. BEND 1 of the existing transfer line will have to be moved slightly or be modified to accommodate the new beam pipe.
The trajectory is constructed by Horizontal and Vertical bending magnets and tangential drift lines. See Figure 5. The bending magnets angles and their desired fields are summarized in Table 1.

Table 1: Summary of the bending magnets needed for CESR IV transfer line

\begin{tabular}{|l|c|c|c|c|}
\hline $\begin{array}{l}\text { Bending } \\
\text { magnet }\end{array}$ & Length & $\begin{array}{c}\text { Bending } \\
\text { angle } \\
\text { (m) }\end{array}$ & $\begin{array}{c}\text { Magnetic } \\
\text { field } \\
\text { (Tesla) }\end{array}$ & $\begin{array}{c}\text { Total } \\
\text { current } \\
\text { (KA-t) }\end{array}$ \\
\hline HSWT & 1.53 & 9.04 & 1.821 & 18.41 \\
V100 & 1.53 & -2.54 & 0.512 & 5.176 \\
V300 & 1.53 & 2.54 & 0.512 & 5.176 \\
HB10 & 1.53 & 8.37 & 1.686 & 1.704 \\
HB20 & 1.53 & 4.34 & 0.874 & 8.835 \\
dc septum & 1.65 & -1.36 & 0.254 & 2.569 \\
p. septum & 1.50 & -2.24 & 0.461 & 4.668 \\
\hline
\end{tabular}

At the input of the transfer line the TWISS parameters are set identical to those of the synchrotron ring at S133, while the TWISS parameters at the output of the transfer line are matched to those of CESR IV at Q051 by varying both the strength and longitudinal position of the quadrupoles. This match of the optics is done by using the optimization program DIM AT [4] with the constraints that the quadrupoles strength be less than $0.6 \mathrm{~m}^{-2}$ and $\beta$ less than $80 \mathrm{~m}$. The variations of the TWISS parameters along the transfer line are seen in Figures $1-4$ with the corresponding quadrupole strengths in Table 2 . Note, the strength and location of QT01 and QT02 which are shared by the two transfer lines were set for the present CESR and have not been changed.

Table 2: Quadrupoles strengths optimized to match input to CESR IV

\begin{tabular}{|l|c|c|}
\hline Quadrupole & Length (m) & Optimized strength \\
\hline Q306 & 0.8 & 0.5837 \\
Q100 & 0.8 & -0.3584 \\
Q200 & 0.8 & -0.2538 \\
Q304 & 0.8 & 0.4926 \\
Q300 & 0.8 & -0.1680 \\
Q303 & 0.8 & 0.0721 \\
Q305 & 0.8 & -0.2423 \\
\hline
\end{tabular}

\footnotetext{
${ }^{*}$ Work supported by NSF.
} 


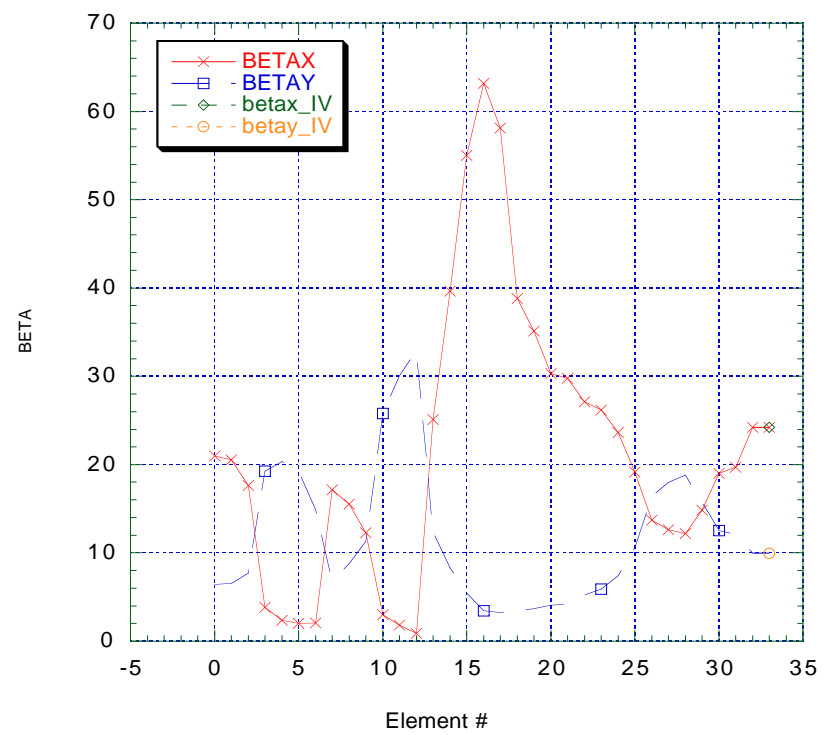

Figure 1: Variations of $\beta$ along CESR IV transfer line. The vertical section is constructed by elements $10-18$

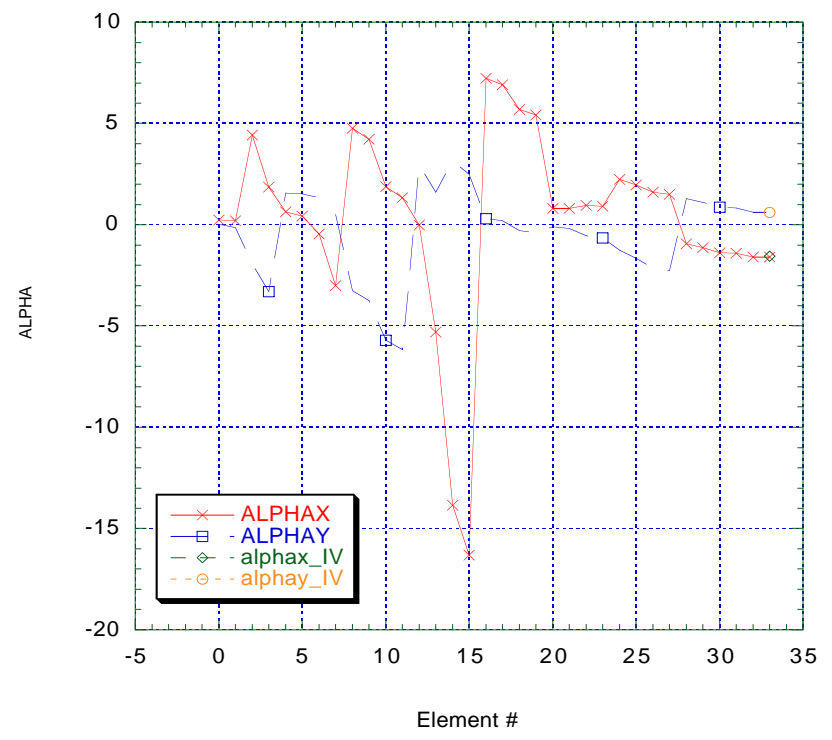

Figure 2: Variations of $\alpha$ along CESR IV transfer line. The vertical section is constructed by elements $10-18$

\section{CONCLUSION}

The total length of the transfer line will be $35.765 \mathrm{~m}$. The obtained TWISS parameters at the output of the transfer line match those of CESR IV well except those of $\eta^{\prime}$. Diagnostic components have not been included yet.

\section{ACKNOWLEDGMENT}

I would like to thank Scott Chapman for supplying the the survey measurements of the synchrotron and the present transfer line.

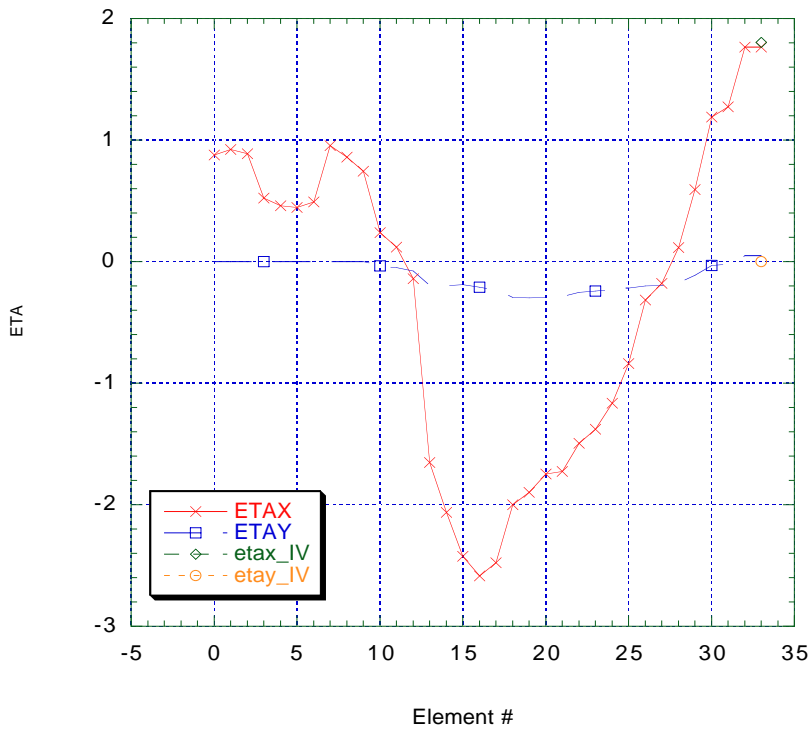

Figure 3: Variations of $\eta$ along CESR IV transfer line. The vertical section is constructed by elements $10-18$

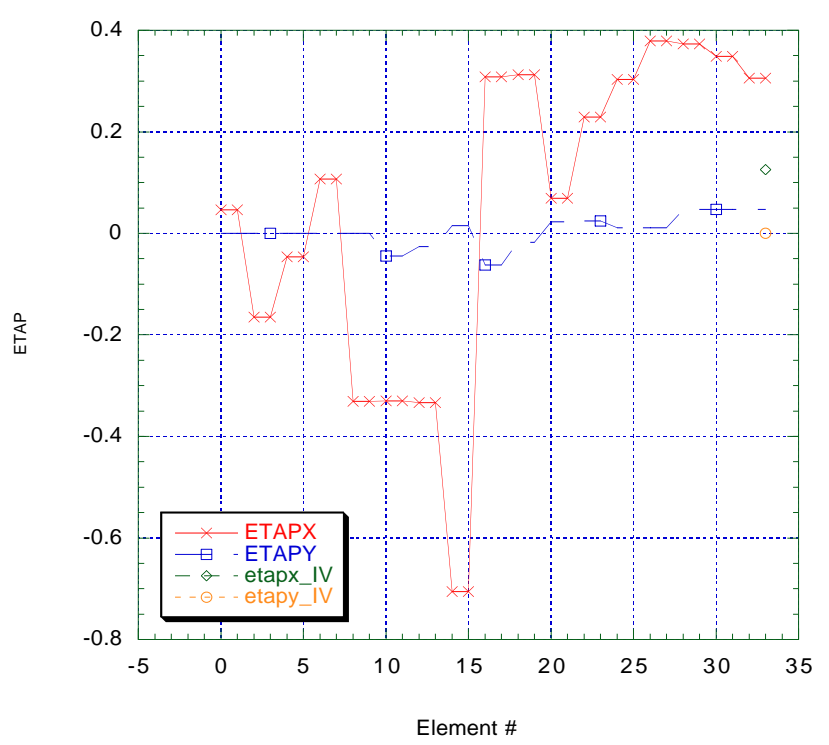

Figure 4: Variations of $\eta^{\prime}$ along CESR IV transfer line. The vertical section is constructed by elements $10-18$

\section{REFERENCES}

[1] G. Dugan, A. Mikhailichenko, J. Rogers, D. Rubin, "Dual Aperture Luminosity Colider at Cornell University," PAC 1997.

[2] J. T. Seeman, 'Injection Process of the Cornell electron Storage Ring CESR," thesis 1979.

[3] S. Chapman, "Survey Measurments of the Synchrotronprivate communication".

[4] R. V. Servranckx, K.L. Brown,'DIMAT”, SLAC report 270 UC-28 (A) 1984. 

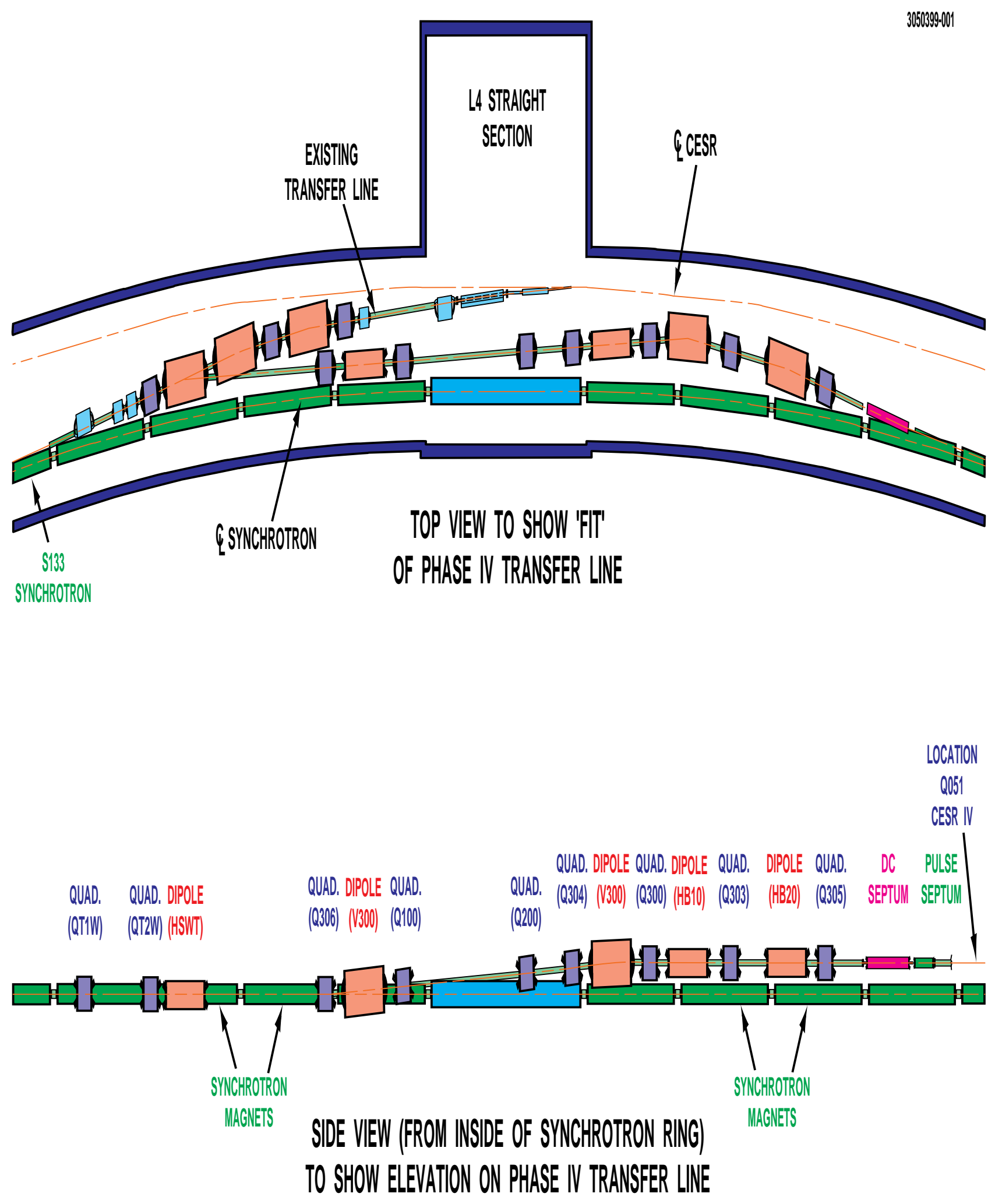

Figure 5: Top and side views of the proposed transfer line for CESR IV. 